\title{
Proyecto Ascendere: Un ecosistema de prácticas de Innovación Docente en la UTPL.
}

Angela del Cisne Salazar Romero ${ }^{a}$, Ana María Beltrán Flandoli ${ }^{\mathrm{b}}$ y María Isabel Loaiza Aguirre ${ }^{\mathrm{c}}$

aacsalazar@utpl.edu.ec, ${ }^{\mathrm{b}}$ ambeltran@utpl.edu.ec y ${ }^{\mathrm{c}}$ miloaiza@utpl.edu.ec.

\begin{abstract}
This Communication presents an approach to concepts of different authors on innovation, as a preliminary analysis that leads to the experiential exhibition detailing the work developed in educational innovation of the Technical University of Loja through the "Ascendere Project".

This project is an initiative that welcomes those efforts that enhance the teaching skills of teachers through academic innovation and research into new methods of education and use of ICT.
\end{abstract}

Keywords: academic_innovation, academy, higher_education, ascendere, UTPL.

\begin{abstract}
Resumen
La presente comunicación recoge una aproximación a conceptos de diferentes autores sobre la innovación docente; esto, a manera de análisis previo que dará pie a la exposición experiencial del trabajo desarrollado en el campo de la innovación docente en la Universidad Técnica Particular de Loja a través del "Proyecto Ascendere".

El mencionado proyecto es una iniciativa que acoge aquellos esfuerzos que potencian las competencias pedagógicas de los docentes a través de la innovación académica y la investigación en nuevas metodologías de educación y uso de las TIC.
\end{abstract}

Palabras clave: innovación_docente, academia, educación_superior, formación, ascendere, UTPL.

\section{Introducción}

El Informe Horizonte 31 (2015); indica que para los siguientes 15 años, el panorama de la educación superior en Iberoamerica avisora una serie de transformaciones categóricas en el desarrollo de su modelo educativo; este cada vez más volcado a la formación y búsqueda del talento humano, perfilará la misión, nicho de acción y gestión de este tipo de instituciones. 
Debido a la clase de cambios sucedáneos que transforman el proceso de enseñanza aprendizaje, los esfuerzos por un perfil docente aún más pertinente y de alto nivel en las universidades debe sostenerse en dos pilares esenciales: por un lado la inminente socialización del conocimiento, con sus vertiginosas formas de presentación a los profesionales en formación de hoy en día; y por otro, la constitución de los profesores como catalizadores de un proceso de cambio, hábiles guías en la búsqueda de este conocimiento, con dominio de las herramientas y metodologías para este fin y sobre todo, inspiradores por su actitud emprendedora y creativa.

Las Universidades por tanto deben trabajar en propuestas sólidas de innovación y formación docente, para empatar los currículos con las tendencias de vanguardia en este sentido, potenciando iniciativas sutantables que serán realizadas y avaladas por la propia comunidad.

De esta manera el presente trabajo aborda dos fases: un marco teórico, con conceptos de diferentes autores sobre el papel de la innovación y sus objetivos, así como el concepto de buenas prácticas en este ámbito, centrado en la docencia universitaria. Y seguido a esto, se presentará una exposición experiencial, en la cual se detalla el trabajo realizado en el campo de la innovación docente ejecutada en la Universidad Técnica Particular de Loja a través del "Proyecto Ascendere - UTPL".

\section{Referentes teóricos y aproximación a las condiciones para la innovación docente universitaria}

\subsection{Algunas definiciones de innovación educativa}

La pauta de una diferenciación estratégica, que sea propia de las particularidades del entorno educativo y que genere las condiciones necesarias para la viabilidad de los proyectos de índole organizativa y académica, constituyen a priori el concepto de innovación educación planteado por Imbernón (1996: 64). Según este autor, para que las innovaciones surtan el efecto deseado, es necesario que su proceso se desarrolle de manera integral y no sesgadamente o por escaños. Es importante mencionar que dentro de su definición, la parte medular de la innovación es contribuir con el mejoramiento social proponiendo respuestas a carencias/falencias presentes en el entorno.

Por su parte Morin y Seurat (1998), definen a la innovación como «el arte de aplicar, en condiciones nuevas, en un contexto concreto y con un objetivo preciso, las ciencias, las técnicas, etc.», considerando que el proceso de innovación no pertenece únicamente al estadio de investigación, donde se obtienen datos y condiciones ideales, sino también a la asimilación por parte de la (nueva) organización, de una tecnología dominada y aplicada eventualmente en otros campos de actividad con propósitos específicos y muy bien deliniados. Así pues, en el ámbito educacional cualquier proyecto que implique cambios metodológicos y la respectiva adaptación, para el caso concreto de nuestra exposición de los profesores universitarios, constituiría una innovación.

(c) EY-NC-ND 2016, Universitat Politècnica de València

Congreso IN-RED (2016) 
Muy clara es la perspectiva de los últimos autores, al declarer que es uso de una la tecnología per se como fuente de innovación, es recién la punta del iceberg; equilibrándose más bien con la generación de nuevas destrezas, resilientes comportamientos y sobretodo con la adopción de prácticas asociadas al cambio por parte de los docentes como principio activo de la institución educativa.

Sobre el tema del cambio, en discusión por algunas decadas, Havelock y Huberman (1980) nos indican diferentes enfoques: desde una perspectiva funcional, la innovación se puede entender como la incorporación de una idea, práctica o artefacto novedoso dentro de un conjunto, con la convicción de que el todo cambiará a partir de las partes que lo constituyen; el cambio se generaría en determinadas esferas y luego se diseminaría al resto del sistema.

Y, desde otra perspectiva menos reduccionista, podemos considerar la innovación como una forma creativa de selección, organización y utilización de los recursos humanos y materiales; forma ésta, nueva y propia, que dé como resultado el logro de objetivos previamente marcados. Estamos hablando, pues, de cambios que producen mejora, cambios que responden a un proceso planeado, deliberativo, sistematizado e intencional. Este proceso complejo, supondría la conjunción de hechos, personas, situaciones e instituciones, actuando en un período de tiempo en el que se dan una serie de acciones para lograr el objetivo propuesto (Havelock y Huberman, 1980).

Como cualquier organización que pretende la calidad, la Universidad, para llevar a cabo verdaderos cambios y verdaderos procesos de innovación, debe prestar, en primer lugar, atención al entorno y sus mensajes. Los cambios que están afectando a las instituciones de educación superior no pueden entenderse sin hacer referencia al contexto de cambios que ocurren en distintos órdenes y que constituyen esa presión externa (Salinas, 2004).

Es conveniente finalizar este apartado anotando el concepto de Jaume Carbonell en su libro "La Aventura de Innovar", quién señala que la innovación educativa sería:

"El conjunto de ideas, procesos y estrategias, más o menos sistematizados, mediante los cuales se trata de introducir y provocar cambios en las prácticas educativas vigentes. La innovación no es una actividad puntual sino un proceso, un largo viaje o trayecto que se detiene a contemplar la vida en las aulas, la organización de los centros, la dinámica de la comunidad educativa y la cultura profesional del profesorado. Su propósito es alterar la realidad vigente, modificando concepciones y actitudes, alterando métodos e intervenciones y mejorando o transformando, según los casos, los procesos de enseñanza y aprendizaje. La innovación, por tanto, va asociada al cambio y tiene un componente - explícito u oculto- ideológico, cognitivo, ético y afectivo. Porque la innovación apela a la subjetividad del sujeto y al desarrollo de su individualidad, así como a las relaciones teoríapráctica inherentes al acto educativo."

(c)) EY-NC-ND 2016, Universitat Politècnica de València

Congreso In-Red (2016) 


\subsection{Innovación en la Universidad}

Las Instituciones de Educación Superior juegan un rol fundamental en el avance científicotecnológico y en esa área son pilares del cambio, sin embargo en el ámbito de la metodología docentes y su adaptación a los nuevos entornos, las Instituciones de Educación Superior en Latinoamérica por ejemplo, han demostrado poca capacidad de adaptación y tienden más bien a ser instituciones conservadoras y con un enfoque tradicionalista.

El nuevo papel de la Universidad ante la sociedad requiere nuevas actitudes y valores, nuevos compromisos y relaciones, nuevas prácticas de cooperación y de servicios, exige nuevas estructuras, nuevas formas de enseñanza-aprendizaje, nuevas modalidades de educación y nuevas concepciones curriculares.

Toffler (1985) señalaba que las organizaciones complejas, y las Universidades lo son, cambian significativamente cuando se dan tres condiciones: presión externa importante, personas integrantes insatisfechas con el orden existente y una alternativa coherente presentada en un plan, modelo o visión.

La complejidad del proceso entonces, se caracteriza por el hecho de introducir cambios sustanciales en los sistemas educativos, porque estos implican nuevas formas de comportamiento y una consideración diferente de los alumnos. Requerirán, por lo tanto, un órden y la ejecución de algunas etapas: sistematización, formalización, seguimiento y evaluación.

Acotando sobre estas etapas Curry (1992), por ejemplo, habla de tres procesos fundamentales: movilización, por la que el sistema es preparado para el cambio; implantación, en la cual el cambio es introducido; e institucionalización, cuando el sistema se estabiliza en la nueva situación.

Para la presente comunicación se define la innovación educativa como la competencia o capacidad institucional disponible para transformar las acciones formativas y organizativas, generadoras y transformadoras de valores y conocimientos a través de la renovación planificado de procesos (Hurtado y Terrón, 2005).

\subsection{Buenas prácticas}

Las organizaciones e instituciones de educación superior que obtienen resultados de calidad poseen claros objetivos estratégicos y significativos, aprovechan sus recursos y talentos y mejoran continuamente a través de procesos sistemáticos de aprendizaje y reflexión.

El concepto de buenas prácticas se utiliza en una amplia variedad de contextos para referirse a las formas óptimas de ejecutar un proceso, que pueden servir de modelo para otras organizaciones.

Para comprender el alcance de la buena práctica docente, Rodríguez (2012) lista una serie de indicadores que resaltan su potencial: primero, las buenas prácticas permiten la generación de un aprendizaje significativo aplicable a la vida diaria. Segundo, involucran a los alumnos en las actividades de aprendizaje ya que trabajar con buenas prácticas requiere una mayor cantidad de operaciones mentales, trabajo colaborativo y estrategias de

(cc) BY-NC-ND 2016, Universitat Politècnica de València

Congreso IN-RED (2016) 
autoaprendizaje autónomas. Tercero, las buenas prácticas promueven el pensamiento divergente. Cuarto, en las buenas prácticas intervienen diferentes campos de conocimiento (interdisciplinariedad y transversalidad), de igual forma establecen interconexiones entre los actores del proceso educativo y también entre grupos de trabajo e instituciones educativas. Finalmente, las buenas prácticas favorecen el uso de las TIC y exigen una evaluación continua.

Como señalan Durán y Estay (2016) independiente de la forma como se aborden, las buenas prácticas no resultan ajenas a ningún sector productivo y son aplicables en el plano individual, institucional u organizacional. Cabe destacar, que los estudios realizados sobre buenas prácticas en el sector educativo deberían compartirse, difundirse e instrumentarse con el propósito de alcanzar los objetivos de calidad y equidad que requiere la universidad.

\section{Objetivos de la Innovación}

Los objetivos que se persigue con el "Proyecto Ascendere" como pilar de la Innovación Docente en la UTPL son:

1. Mejorar la calidad educativa de las titulaciones de grado y postgrado.

2. Generar movilidad docente hacia otras universidades con las cuales se pueda compartir las experiencias de las buenas prácticas docentes.

3. Crear espacios para identificar, valorar, sistematizar, normalizar, aplicar y difundir las experiencias novedosas que contribuyan a la solución de problemas educativos que estén afectando la calidad de los aprendizajes.

4. Estimular la investigación como un elemento cotidiano determinante de la formación profesional continua de los docentes a partir de su propia práctica educativa.

5. Documentar las experiencias de las buenas prácticas docentes innovadoras.

6. Compartir y transferir a otros docentes las experiencias de buenas prácticas docentes innovadoras para ampliar y generalizar la experiencia.

7. Crear condiciones permanentes para que las experiencias innovadoras se conviertan en una práctica institucionalizada, es decir, en cultura organizacional.

8.

\subsection{Acercamiento contextual:}

Proyecto Ascendere: Un ecosistema de prácticas de Innovación Docente en la UTPL.

Con base a la necesidad de reunir el complejo sistema de iniciativas de innovación e investigación educativa que se realizan en la Universidad Técnica Particular de Loja, las mismas que al ser ejecutadas por diferentes colectivos de esta institución: grupos interdisciplinares, instancias gestoras de política académica, áreas y departamentos académicos, etc; deben asociarse de forma estratégica para promover un accionar conjunto; se impulsó a finales del año 2015 la creación del Proyecto Ascendere, una iniciativa que

(c)) EY-NC-ND 2016, Universitat Politècnica de València

Congreso In-Red (2016) 
acoge aquellos proyectos que potencian las competencias pedagógicas de los docentes a través de la innovación y formación académica y la investigación en nuevas metodologías de educación y uso de las TIC.

El nombre que da vida al proyecto, evoca la frase "memento ascendere semper" (recuerda superarte siempre), que se encuentra plasmada en el escudo de la institución. El objetivo es reafirmar el compromiso de la UTPL de contar con docentes que inspiren a sus alumnos y formen en ellos un espíritu innovador vinculado con la práctica de su profesión y que se encuentre a la vanguardia de su disciplina y del mundo.

Este proyecto, que se desarrolla en la Dirección de Innovación, Formación y Evaluación Docente del Vicerrectorado Académico de la UTPL, se lanza con el objetivo de comunicar efectivamente las diferentes actividades y potenciar a la innovación docente en su amplia tipología.

Algunas de las iniciativas dirigidas todos a los 1113 docentes que actualmente forman parte de la Universidad son: Laboratorio de Innovación e Investigación Educativa (Liid), Programa de Formación Docente Pedagógica, Encuentro Encuentro InnovaTIC, EduTendencias y la Convocatoria de Proyectos de Innovación Docente, que para el presente trabajo se toma como referente para la comunicación respectiva y la exposición de los resultados alcanzados por nuestra Universidad en el campo de la innovación docente.

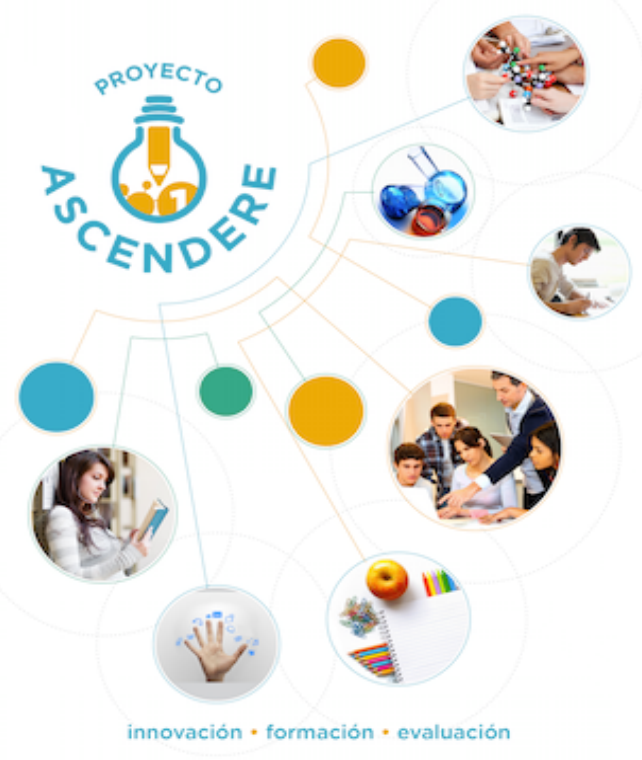

Imagen representativa del "Proyecto Ascendere" Innovación, Formación y Evaluación UTPL 


\section{Desarrollo de la innovación:}

\section{Experiencia en Innovación Docente ejecutada en la Universidad Técnica Particular de Loja a través del "Proyecto Ascendere".}

La Innovación docente (ID) en la Universidad Técnica Particular de Loja, es un proceso coherente, global y original de cambio en la planificación, desarrollo y evaluación de la docencia, lo cual consigue mejorar la formación del estudiante, incluye nuevas formas o alternativas para abordar el proceso de la educación con el fin de lograr mejoras cualitativas y cuantitativas.

Tiene el propósito de cambiar, transformar y mejorar la práctica pedagógica de los docentes mediante la participación activa de todos los miembros de la comunidad educativa. Se pretende una docencia universitaria de calidad, donde la comunicación creativa y eficaz del profesorado con sus estudiantes sea el eje central del proceso.

La innovación, tanto en clases teóricas, prácticas como en tutorías, tiene el objetivo de enriquecer el aprendizaje del estudiante y la adquisición de competencias profesionales. La docencia universitaria debe incorporar problemas y necesidades reales presentes en la sociedad y nutrirse de los avances de la investigación científica que intenta dar respuestas a dichos problemas y necesidades.

\subsection{Una aproximación del desarrollo de la ID; desde la perspectiva y funcionalidad de} la Convocatoria de Buenas Prácticas Prácticas en Innovación Docente de la UTPL.

El Plan Estratégico de Desarrollo Institucional 2011-2020, enuncia entre sus líneas estratégicas la formación en la docencia pertinente y de alto nivel, señalando la importancia y el compromiso que tiene la institución con la formación integral de los estudiantes y del personal de la UTPL. Para ello, es esencial motivar y apoyar la innovación, el emprendimiento y las buenas prácticas en el desempeño de las actividades docentes.

Con esta finalidad, semestralmente se hace pública la octava convocatoria para participar con las propuestas de buenas Prácticas Docentes.

Esta convocatoria es una invitación a todos los docentes de la Universidad para que participen e implementen nuevas estrategias de enseñanza aprendizaje, potenciando el uso creativo de diferentes herramientas dentro y fuera del aula, implicando a los estudiantes activamente en su proceso de aprendizaje, planificando, desarrollando y evaluando las competencias necesarias para contribuir con el perfil de egreso de la titulación e incorporando además en el desarrollo de la docencia problemas reales del entorno.

Además, por medio de la convocatoria se intenta compartir las experiencias de las buenas prácticas de los docentes y estudiantes a nivel interno en la universidad, incentivar y apoyar la participación de los docentes en Congresos de relevancia nacional e internacional para difusión de las mismas. 


\subsubsection{Lineas estratégicas para presentación de proyectos de innovación en la docencia}

Los profesores de Modalidad Presencial, Abierta y de Postgrados presentan propuestas de Buenas Prácticas Docentes, enmarcadas en la implantación de estrategias de enseñanzaaprendizaje, que potencian el uso creativo de diferentes herramientas dentro y fuera del aula. Para la presentación de proyectos de innovación en la docencia, se consideran las siguientes líneas estratégicas:

\section{- Proyectos de innovación educativa con uso de REAS}

Los Recursos Educativos Abiertos o REA (en inglés Open Educational Resources, OER) son documentos o material multimedia con fines relacionados con la educación como la enseñanza, el aprendizaje, la evaluación y la investigación cuya principal característica es que son de acceso libre y por lo general bajo licencia abierta.

Con estos proyectos se busca fomentar el uso de materiales y recursos educativos que puedan ser reutilizados para la docencia y el aprendizaje de forma gratuita.

\section{- Proyectos para crear observatorios universitarios}

Proyectos que contribuyan para realizar un levantamiento de información y que permitan obtener líneas bases de temas determinados, con estos proyectos se pretende tener un repositorio donde los profesionales, directores, estudiantes y docentes encuentren resultados y temas de interés para fortalecer sus trabajos.

\section{- Proyectos de innovación en actividades académicas y de gestión docente}

Se consideran proyectos de actividades académicas y de gestión docente aquellos en los que participen varios profesores de una o más secciones departamentales con la finalidad de que presenten propuestas que estén orientadas a mejorar el rendimiento académico de los estudiantes.

\section{- Proyectos mentores docentes-estudiantes}

El proyecto mentores docentes-estudiantes procura facilitar el proceso de integración a la Universidad de los estudiantes de nuevo ingreso de la Modalidad Presencial, a través de actividades de acompañamiento permanente.

\section{- Proyectos de aprendizaje basado en juegos (Gamification)}

Proyectos que vayan dirigidos al aprendizaje basado en la utilización de mecanismos y técnicas a través de juegos que motiven la participación de los estudiantes, para que por medio de su estructura proveniente de los juegos logre su aprendizaje y adquiera las competencias. Se puede diseñar juegos con herramientas tecnológicas o físicas.

\section{- Proyectos de aprendizaje con metodología Flipped Classroom (FC)}

El Flipped Classroom (FC) es un modelo pedagógico que transfiere el trabajo de determinados procesos de aprendizaje fuera del aula y utiliza el tiempo de clase, junto con la experiencia del docente, para facilitar y potenciar otros procesos de adquisición y práctica de conocimientos dentro del aula.

(c) EY-NC-ND 2016, Universitat Politècnica de València 
- Proyectos de Innovación en metodologías docentes para clases teóricas y prácticas

Proyectos dirigidos a la innovación en: clases magistrales, estudio de casos prácticos, resolución de ejercicios y problemas, aprendizaje basado en problemas, aprendizaje por proyectos, aprendizaje cooperativo, clases prácticas.

\section{- Proyectos de Innovación en la evaluación de los estudiantes}

Proyectos dirigidos a la puesta en práctica de nuevos instrumentos para la evaluación de los estudiantes, que propicien un aprendizaje significativo y sostenido, y la evaluación de la adquisición de competencias.

\section{- Proyectos de Innovación en enseñanza bilingüe}

Proyectos que fomente y apoyen el aprendizaje de una segunda lengua-

\section{- Proyectos de Innovación en la Gestión Productiva}

Proyectos dirigidos a la innovación en recursos docentes y estrategias educativas que permitan la adquisición de competencias profesionales relacionando la teoría con la práctica.

\section{- Proyectos de Innovación Social y/o Emprendimiento Social}

Se consideran innovaciones de este tipo aquellos proyectos que generen un impacto y una ayuda social, impulsando actividades de emprendimiento social relacionando las actividades académicas de los docentes y estudiantes con las necesidades del entorno.

\section{- Proyectos de Innovación para mejorar la formación de estudiantes con necesidades educativas especiales}

Proyectos que involucren actividades que apoyen y mejoren la formación de estudiantes con necesidades educativas especiales.

\section{- Proyectos de Innovación en la gestión On-line de los procesos de enseñanza aprendizaje}

Proyectos dirigidos a la elaboración y mejora de materiales docentes innovadores en la Plataforma del Entorno Virtual de Aprendizaje (EVA), que integren herramientas web de ayuda a la docencia proyectos que propongan la creación de materiales multimedia (podcasts, vídeos, animaciones, etc.) o materiales audiovisuales.

\section{- Proyectos con Aplicación de Tecnologías de la Información y Comunicación (TICs)}

Se consideran innovaciones en las cuales se propicie el uso sistemático de herramientas tecnológicas, ya existentes, para la mejora del proceso de enseñanza-aprendizaje (Internet, multimedios, videos, software educativo, simuladores, plataformas virtuales, portafolios electrónicos, etc.) 


\section{- Proyectos profesores mentores- noveles}

En esta línea lo fundamental es ofrecer apoyo al profesor de nuevo ingreso, entendiéndose como este a aquel que inicia su carrera en la vida universitaria y que tienen tres años o menos de ejercicio de la docencia (profesor novel), a través de un seguimiento a su actividad docente de manera integral y personalizada por parte de los profesores antiguos o con experiencia en el manejo de la actividad académica denominados mentores.

\section{- Proyectos ayudantes de cátedra}

La necesidad de apoyo a la docencia en la Universidad Técnica Particular de Loja (UTPL) conlleva a que los estudiantes de pregrado puedan realizar tareas de apoyo a la docencia en calidad de ayudantes de cátedra.

\section{- Proyectos de innovación en la docencia de postgrados}

Proyectos dirigidos a innovar y mejorar la planificación, desarrollo y evaluación en las actividades docentes de postgrado.

\subsubsection{Criterios para la aprobación de los proyectos}

Los criterios que se establecieron para la aprobación de los proyectos presentados son los siguientes:

- Grado de la innovación y claridad en la formulación que presente la propuesta

- Metodología y tareas concretas a desarrollar para implantar la buena práctica de innovación docente.

- Coherencia con las líneas estratégicas definidas en esta convocatoria.

- Relevancia y pertinencia de la innovación para mejorar la práctica docente.

- Viabilidad económica y pedagógica de las propuestas.

- Incidencia en la mejora de la práctica docente con repercusión en los procesos de aprendizaje de los estudiantes.

- Igualmente, se valorará positivamente aquellos que se comprometan expresamente a dar difusión de su actividad en congresos, revistas especializadas, foros u otros medios.

\subsubsection{Desarrollo y seguimiento}

Una vez aprobado el proyecto de innovación se comunica la resolución a los docentes responsables de la postulación de la Buena Práctica Docente, los proyectos se implementan durante el período académico abril-agosto 2016. Al finalizar el proyecto y en un plazo máximo de un mes, los equipos de profesores presentan un informe final, el cual adopta el formato del anexo 1. En el informe se debe reflejar los resultados de la buena práctica, los resultados académicos de los estudiantes y evidencias del desarrollo de la buena práctica (fotos, gráficos, etc.).

Así mismo se aplica una encuesta a los estudiantes con la finalidad de medir el desarrollo de la buena práctica docente y la contribución que ha tenido en el aprendizaje del estudiante. 


\section{Resultados de la aplicación de la Convocatoria de Buenas Prácticas de Innovación Docente como experiencia de Innovación Docente en la UTPL dentro del "Proyecto Ascendere".}

Un total de 151 docentes interesados en potenciar el uso creativo de herramientas, dentro y fuera del aula, que permitan que los estudiantes participen activamente en el proceso de aprendizaje, adquieran las competencias y el perfil de egreso de su titulación, y se incorporen en la solución de problemas reales en el entorno; se involucraron en 50 proyectos de innovación pedagógica a través de dos convocatorias en el año 2015. Los mejores proyectos fueron presentados como buenas prácticas en las Jornadas de Reflexión Académica para conocimiento e inspiración del claustro docente, y posteriormente se reconoció a los diez docentes más innovadores del año 2015 de las diferentes áreas académicas. Para el año 2016

También es esencial dar un espacio para dar a conocer parte de otras iniciativas que forman parte del "Proyecto Ascendere", 19 docentes participaron en el Modelo de Cátedra Compartida en 12 componentes académicos, y 21 estudiantes de las diferentes titulaciones en los dos periodos académicos, fueron parte del proyecto Ayudantes de Cátedra. Adicional a ello, se realizaron diez encuentros "Café Científico" que reunieron a académicos y profesionales para establecer diálogos sobre temas de actualidad encaminados a la formulación de proyectos en conjunto, contando con la participación aproximada de 350 docentes.

Tabla 1. Histórico de partipación en la Convocatoria de Buenas Prácticas Docentes Períodos: año 2012 al 2016

\begin{tabular}{|l|l|l|l|l|l|}
\hline $\begin{array}{l}\text { Período } \\
\text { académico }\end{array}$ & $\begin{array}{l}\text { Nro. de } \\
\text { proyectos }\end{array}$ & Presencial & Distancia & $\begin{array}{l}\text { Nro. De } \\
\text { docentes }\end{array}$ & $\begin{array}{l}\text { Nro. De } \\
\text { estudiantes * }\end{array}$ \\
\hline $\begin{array}{l}\text { ctubre 2012- } \\
\text { febrero 2013 }\end{array}$ & 13 & 11 & 2 & 36 & 752 \\
\hline Abril-agosto 2013 & 23 & 20 & 3 & 42 & 1475 \\
\hline $\begin{array}{l}\text { Octubre 2013- } \\
\text { febrero 2014 }\end{array}$ & 16 & 13 & 3 & 39 & 1035 \\
\hline Abril-agosto 2014 & 9 & 9 & 0 & 23 & 980 \\
\hline $\begin{array}{l}\text { Octubre 2014 - } \\
\text { febrero 2015 }\end{array}$ & 19 & 15 & 4 & 58 & 1380 \\
\hline
\end{tabular}

(cc) EY-NC-ND 2016, Universitat Politècnica de València

Congreso In-Red (2016) 


\begin{tabular}{|l|l|l|l|l|l|}
\hline Abril-agosto 2015 & 19 & 14 & 5 & 51 & 1345 \\
\hline $\begin{array}{l}\text { Octubre 2015 - } \\
\text { febrero 2016 }\end{array}$ & 31 & 26 & 5 & 84 & 1975 \\
\hline & $*$ Participan estudiantes de Modalidad Presencial y a Distancia \\
\hline
\end{tabular}

Tabla 2. Datos relevantes de la Convocatoria de Buenas Prácticas de Innovación Docente. Períodos: año 2012 al 2016.

\begin{tabular}{|l|}
\hline DATOS RELEVANTES \\
\hline $\begin{array}{l}\text { Se han realizado } 7 \text { convocatorias de buenas } \\
\text { prácticas de innovación docente }\end{array}$ \\
\hline $\begin{array}{l}\text { Los docentes participantes han divulgado las } \\
\text { buenas prácticas en Congresos en España, chile, } \\
\text { Colombia, Argentina y México }\end{array}$ \\
\hline $\begin{array}{l}\text { A partir de las experiencias en la Convocatoria se } \\
\text { ha logrado la publicación de } 6 \text { artículos en } \\
\text { revistas indexadas o de divulgación científica. }\end{array}$ \\
\hline
\end{tabular}

También es importante mencionar que para visibilizar estas y otras actividades que promueven la creatividad, innovación y uso eficaz de las TIC para potenciar la calidad académica, adaptándose a las necesidades y requerimientos del siglo XXI, se creó el portal web del Proyecto Ascendere: innovaciondocente.utpl.edu.ec

\section{Conclusiones}

Los procesos de innovación docente involucran cambios y la participación de diferentes actores: estudiantes, docentes, autoridades; así como, cambios de metodologías de enseñanza aprendizaje, incorporación de nuevas estrategias de colaboración, planes de formación continua a los docentes, incremento de recursos, pero sobretodo la motivación que debe estar presente en los actores para innovar.

La innovación va ligada de las actividades de investigación que se desarrollan en las IES, no puede haber innovación sin investigación, investigar para innovar, conocer las necesidades del entorno y formar a los estudiantes para que den respuesta a un mundo que está en continuo cambio es responsabilidad de una universidad innovadora.

Actualmente en el contexto latinoamericano y particularmente el de nuestra institución la UTPL, el rol que desempeña el docente es muy amplio, ya que ha pasado de ser la única fuente del conocimiento a ser un referente más de información, por lo que su formación 
debe estar preparada para ser un facilitador, un guía para los estudiantes. Además de transmitir conocimientos, teorías, conceptos, les ayuda a desarrollar habilidades y talentos y por medio de su ejemplo de vida, de sus propias vivencias y de su personalidad, contagia de sus buenos sentimientos y emociones que servirán para formar a los estudiantes con actitudes positivas, motivándolos con confianza e integridad a construir su modelo de vida personal y profesional.

\section{Referencias Bibliográficas}

Aragón Revuelta, P. et al. (2015). The changing Marketplace for Higher Educacion. USA: Johns Hopkins Press.

Carbonell, J. (2002). La aventura de innovar. El Cambio en la Escuela. Ediciones Morata.

Curry, B.K. (1992). Instituting enduring innovations: achieving continuity of change in higher education. Washington, DC: ASHE-ERIC Higher Education Reports. https://innovacioneducativa.wordpress.com/2007/01/09/\%C2\%BFque-es-innovacioneducativa/

Duran, R., Estay, C. (2016). Formación en buenas prácticas docentes para la educación virtual. RIED. Revista Iberoamericana de Educación a Distancia, 19 (1), 209-232. doi: http://dx.doi.org/10.5944/ried.19.1.13845 [Consulta: 12 de marzo de 2016].

Imbernón, F. (1996). En busca del Discurso Educativo. Edit. Magisterio del Río de la Plata, Buenos Aires - Argentina.

Hurtado, J., Terrón, A. (2005). El proceso de innovación educativa en la formación permanente del profesorado universitario: Un estudio multicaso.Revista Española De Pedagogía, 63(232), 529-551. Retrieved from http://www.jstor.org/stable/23766333

Luque, T. et al. (2015). Horizon 2031, University of Granada in Lights of its V Centenary "Reflections on te future of the University ». España. Design and layout: Luis Doña Toledo ISBN 978-84-338-5768-2 DL. GR./476-2015. [Consulta 20 de marzo de 2016].

Morin, J..; Seurat, R. (1998). Gestión de los recursos tecnológicos. Madrid: Cotec.

Rodriguez, D.J. (2012). Buenas Prácticas en el ámbito educativo y su orientación a la gestión el conocimiento. Educación, 17 (33), 29 -48

Salinas, J. (2004). "Innovación docente y uso de las TIC en la enseñanza universitaria". Revista de Universidad y Sociedad del Conocimiento (RUSC). [artículo en línea]. UOC. Vol. 1, no 1. [Fecha de consulta: dd/mm/aa]. http://www.uoc.edu/rusc/dt/esp/salinas1104.pdf

Santana Vega, L. (2014). La innovación educativa: Un desafío para los orientadores como agentes promotores de las iniciativas de cambio $=$ The educational innovation: A challenge for the counselors like agents of change. REOP - Revista EspañOla De OrientacióN Y PsicopedagogíA, $21(2)$ 261-270. doi:http://dx.doi.org/10.5944/reop.vol.21.num.2.2010.11530 


\section{ANEXO 1}

FORMATO PARA POSTULACIÓN PREVIA APROBACIÓN DE BUENAS PRÁCTICAS DOCENTES

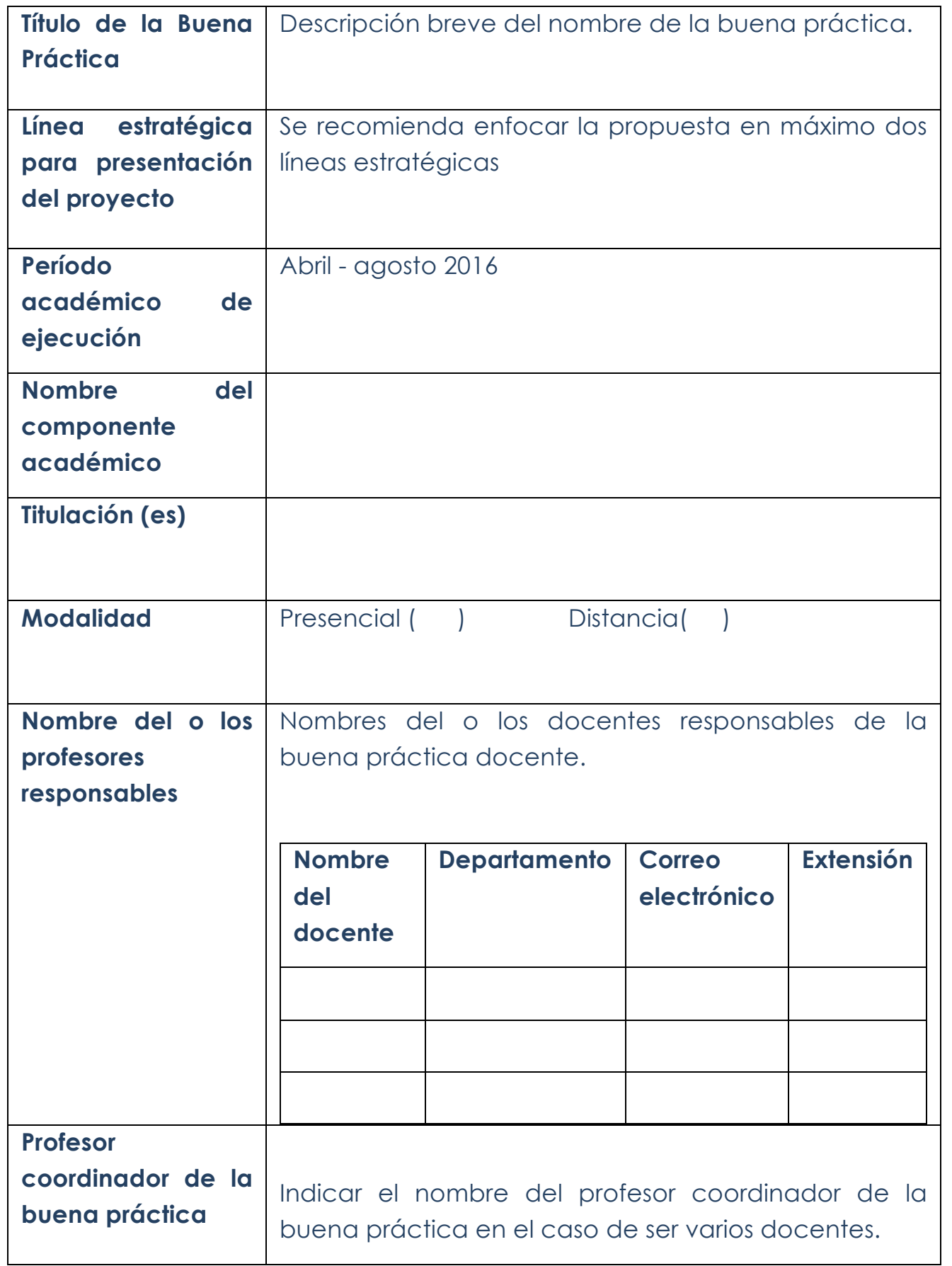




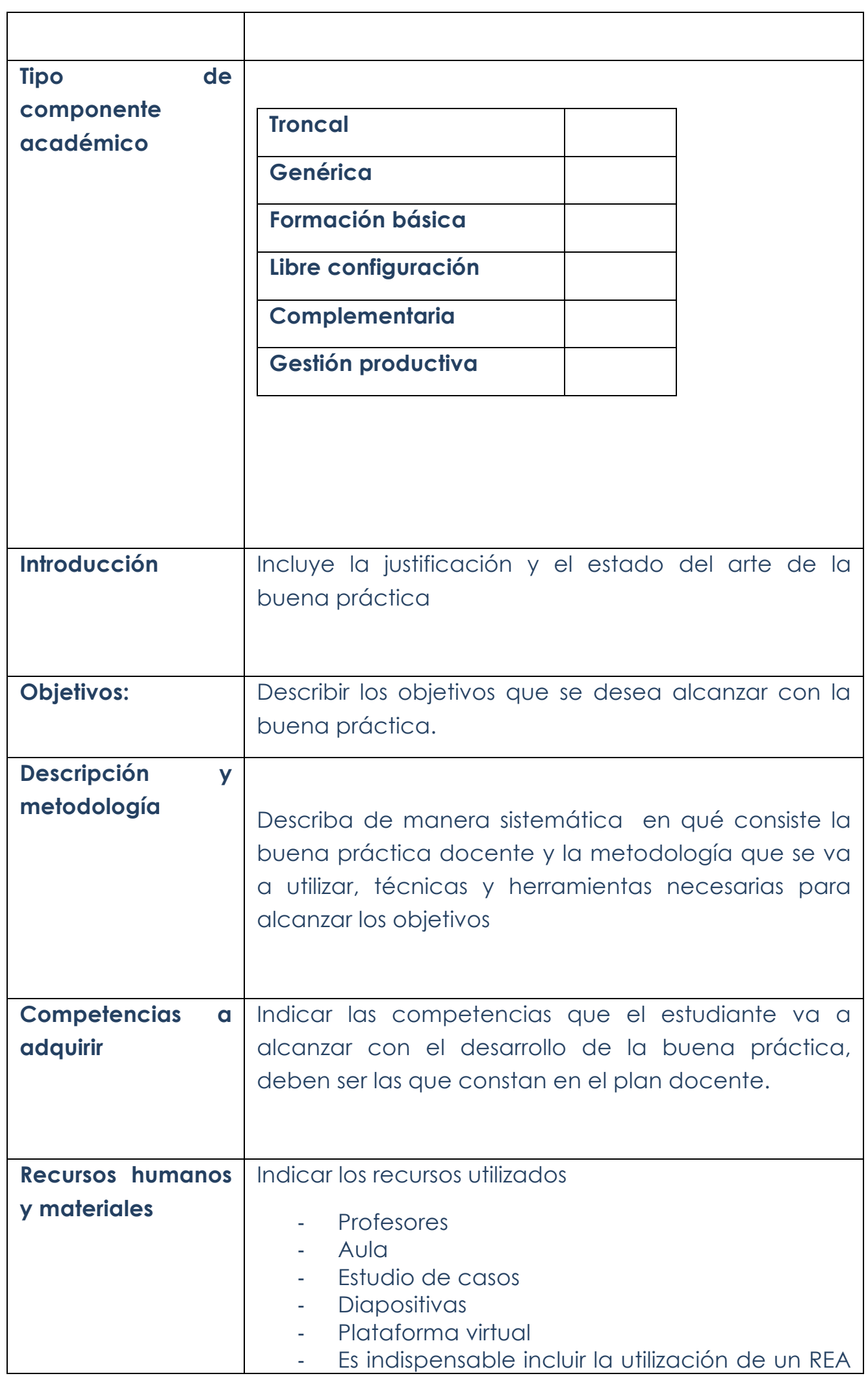

(cc) BY-NC-ND 2016, Universitat Politècnica de València

Congreso In-Red (2016) 
Proyecto Ascendere: Un ecosistema de prácticas de Innovación Docente en la UTPL.

\begin{tabular}{|c|c|}
\hline & (Recurso Educativo Abierto) \\
\hline Presupuesto & $\begin{array}{l}\text { Incluir el presupuesto que requiere para el desarrollo } \\
\text { de la buena práctica. (opcional) }\end{array}$ \\
\hline $\begin{array}{l}\text { Firmas del o los } \\
\text { participantes }\end{array}$ & \\
\hline $\begin{array}{ll}\text { Nombre } & \text { del } \\
\text { Responsable de } & \text { Sección } \\
\text { Departamental } & \end{array}$ & \\
\hline $\begin{array}{ll}\text { Firma } & \text { de } \\
\text { aprobación } & \text { del } \\
\text { Responsable } & \text { de } \\
\text { Sección } & \\
\text { Departamental } & \end{array}$ & \\
\hline
\end{tabular}

